\section{Effectiveness of emergency argon laser retinopexy performed by trainee doctors}

Department of Vitreo-retinal Surgery

Birmingham \& Midland Eye Centre City Hospital NHS Trust, Dudley Road Birmingham, UK

Correspondence: AK Tyagi Birmingham \& Midland Eye Centre City Hospital NHS Trust, Dudley Road Birmingham B18 7 QU, UK Tel: +44121554 3801 Fax: + 441215076853 E-mail: ajaityagi@ yahoo.com

Received: 22 May 2003 Accepted: 29 December 2003

Published online: 16 April 2004

Presented at the Society of European Ophthalmologists meeting (SOE 2003) in Madrid in June 2003.

\begin{abstract}
Objective To report the outcomes following treatment of retinal tears with argon laser photocoagulation by trainee doctors as an emergency procedure.

Methods Retrospective, case note analysis of 100 consecutive patients treated between August 2000 and December 2002 at a tertiary referral hospital.

Result The case notes of $\mathbf{1 0 0}$ consecutive patients (41 male, 59 female) with a mean age of 57.5 years were reviewed. Out of these 90 were symptomatic. The follow-up period ranged from 1 week to 8 months.
\end{abstract}

All patients had argon laser retinopexy in the Emergency department. The on-call Registrar performed the procedure on 94 patients, and Senior House Officers performed the other six. A total of 98 procedures were performed on the slit lamp and two by laser indirect ophthalmoscope. In all, 24 patients needed further treatment with either indirect laser, cryotherapy, or surgery.

At the last follow-up, all the patients had anatomically attached retinas.

Conclusion A significant proportion of patients $(24 \%)$ undergoing laser retinopexy in the emergency department needed further treatment. The relative inexperience in using the indirect laser, together with its unavailability in the Emergency department, may be the contributory factors. There seems to be scope for supervised training in using the laser indirect ophthalmoscope for the trainees in the Ophthalmic Emergency department.

Eye (2005) 19, 52-54. doi:10.1038/sj.eye.6701416

Published online 16 April 2004
YK Ghosh, S Banerjee and AK Tyagi

Keywords: argon laser; laser indirect ophthalmoscope; retinal tear; retinal detachment; retinopexy

\section{Introduction}

Various modalities of treatment have been used for the management of retinal tears to prevent rhegmatogenous retinal detachment. The first methods tried were cautery and diathermy, ${ }^{1}$ which did not prove to be very successful. These were then replaced in the 1950s by the xenon arc photocoagulator. ${ }^{2,3}$ Next to be popularised was cryotherapy. ${ }^{4}$ Argon laser photocoagulation to treat retinal breaks was first reported by Zweng ${ }^{5}$ in the late 1960 s.

Since then, argon laser retinopexy has become widely practised as the first-choice treatment to prevent the occurrence of retinal detachment. ${ }^{6}$ Laser retinopexy treatment for retinal tears needs a certain level of experience and expertise. This is more important for anterior breaks in which indirect laser delivery may be necessary.

There is very little reference in the literature to argon laser retinopexy being performed as an emergency procedure. The aim of this study was to analyse the effectiveness of argon laser retinopexy for retinal breaks, when performed by doctors in training, as an emergency procedure.

\section{Materials and methods}

A retrospective analysis of case notes of all patients undergoing argon laser retinopexy in the Emergency department of Birmingham \& Midland Eye Centre, Burmingham, UK, between August 2000 and January 2003 was undertaken. Patients were identified from the 
recorded database in the laser clinic. All the case notes were then reviewed.

The inclusion criteria were as follows: 1 . Patients with retinal breaks presenting in the emergency department and having laser treatment on the same day; (2) treatment performed by a trainee doctor (specialist registrar or senior house officer); and (3) No previous history of vitreo-retinal surgery performed on the same eye.

We chose to look at 100 consecutive patients because this was considered to be statistically significant.

Preoperative data collection included age and sex of the patient, laterality, lens status, refractive status, presenting symptoms, type and position of the retinal tear, and status of the doctor performing the treatment. We looked at the delivery system preferred for the laser treatment - slit lamp or indirect ophthalmoscope.

Postoperative data were collected for the length of follow-up (whether in speciality clinic), nature of and reason for further treatment in the same eye, and the final anatomical and visual outcome.

The retinopexy procedure varied between argon laser treatment delivered either from the slit lamp or via the indirect ophthalmoscope. The treatment consisted of surrounding the retinal break with two to three rows of confluent white laser burns using either a contact lens or a noncontact condensing lens system. This was noted from the surgical notes. Whether or not the anterior edge of the retinal break had been completely encircled was also noted from the operative observations.

\section{Results}

A total of 122 consecutive cases had emergency laser retinopexy treatment during that period. Complete records were available for 106 patients. Of these, 100 cases were selected, as their treatment had been performed by trainee doctors. The other six had been treated by Associate Specialists and Consultants.

In all, 100 patients (59 female and 41 male) underwent laser retinopexy during the study period. A total of $90 \%$ of the patients were symptomatic and their mean age was 57.5 years (range 27-75 years). Floaters and flashing lights were the most common symptoms in 54 of these patients. The rest presented with either blurred vision (29), reduced visual acuity (5) or a shadow in front of the eyes (2).

The majority of patients (75) had a superior break. Posterior vitreous detachment was noted to be present in 63 patients. In all, 29 patients had associated vitreous haemorrhage.

A total of 94 patients were treated by the on-call Registrar (92 on slit lamp and two with indirect laser). Six were treated by Senior House Officers (SHOs). In this second group, all the six patients were treated on the slit lamp. In all, $51 \%$ of the patients were reviewed in the retina clinic. The mean postoperative follow-up period was 7.3 weeks (range 1 week to 8 months) before being discharged.

Out of the 24 patients requiring retreatment, $16(66.6 \%)$ needed treatment with either the argon laser indirect ophthalmoscope (14) or cryopexy (2). This was done by the vitreo-retinal team. In all, 13 of these patients had inadequate anterior coverage of the retinal break, requiring additional indirect laser. One patient had additional indirect laser, as the original treatment itself was considered to be inadequate. Two patients had large tears unsuitable for laser treatment and required cryotherapy. The remaining eight patients (33.3\%) needed cryopexy and buckle surgery, which were also performed by the vitreo-retinal team. Of these eight patients, three had retinal detachments by the time they were reviewed by the vitreo-retinal team. The other five patients had a large tear with subretinal fluid, which was considered to be unsuitable for laser retinopexy alone and had to have a cryotherapy and external buckling procedure to support it.

The visual acuities of these patients ranged from $6 / 9$ to Count fingers (CF) preoperatively. Postoperatively their visual acuities were noted to be between 6/9 and 6/60. All the patients who required indirect laser or cryotherapy only maintained their visual acuity. Of those patients requiring cryotherapy and external buckling procedure, one patient each lost one and three lines, respectively, on the Snellen's chart due to associated retinal detachment. All the patients had retinal reattachment postoperatively and on their last follow-up visit.

\section{Conclusion}

Argon laser retinopexy is a safe and reliable technique for the treatment of symptomatic flap tears and other retinal breaks that are at a high risk of developing retinal detachment. ${ }^{14}$

The necessity of treating predisposing retinal lesions prophylactically has been documented by various authors. ${ }^{7-9}$ However, prophylactic treatment may not reduce the risk of detachment in eyes with severe myopia or extensive lattice, ${ }^{10}$ as was seen in one of our cases. It also does not eliminate the risk of retinal detachment caused by new retinal breaks occurring outside the prophylactically treated area, ${ }^{10}$ which was also exemplified in one of our cases.

But very little has been written about argon laser retinopexy performed as an emergency procedure in an emergency setting by trainee ophthalmologists. Our 
report highlights this issue and points out the outcomes and problems encountered.

Although the slit-lamp-delivered laser system was more commonly used, it is not suitable for all cases. It is generally accepted that the anterior aspect of the retinal break is by far the most important part to seal, as this is the area that is most subjected to vitreous traction. ${ }^{11}$ Very often, it is this area that is not completely accessible with the slit-lampdelivered laser system. Therefore, use of the laser indirect ophthalmoscope is important in these cases and the trainee doctors need to be more conversant in this technique.

Identifying the difficult cases and seeking early advice from the vitreo-retinal team may help in improving the training standards. Patient selection and performing the laser treatment under supervision would also be a step forward. The follow-up of these patients also needs to be streamlined and a protocol needs to be in place for specialist clinic follow-up and their timing.

The treatment of asymptomatic patients with retinal breaks remains a grey area. However, retinopexy should be considered in eyes with extensive lattices and in nonphakic eyes with fellow-eye abnormality. ${ }^{12,13}$

Argon laser retinopexy is a safe and effective technique for the treatment of symptomatic flap tears and other high-risk retinal breaks. However, this procedure is of limited use in large retinal breaks, opaque media, small and nonreactive pupils, and unsatisfactory tissue response. $^{14}$

We are treating symptomatic retinal breaks as suggested in the reference. ${ }^{15}$

However, a significant proportion of patients (24\%) undergoing laser retinopexy in the Emergency department needed further treatment. The relative inexperience in using the indirect laser together with its unavailability in the Emergency department may be contributing to this effect.

To conclude, there seems to be scope for supervised training in laser indirect ophthalmoscopy and management of retinal breaks, for the Registrars and other junior doctors in the Ophthalmic Emergency department. This report has also prompted an audit of training requirements among the Registrars-intraining, in Laser Indirect Ophthalmoscopy.

\section{References}

1 Preventive treatment of idiopathic and secondary retinal detachment: panel discussion. Chairman, Weve, HJM. XVIII Concilium Ophthalmologicum 1958 Belgica. Brussels: Imprimerie Medicale et Scientifique, SA, 1958; 1: 965-1050.

2 Colyear BH, Pischel DK. Preventive treatment of retinal detachment by means of light coagulation. Trans Pac Coast Otoophthalmol Soc 1960; 41: 193-215.

3 Okun E, Cibis PA. Photocoagulation in limited retinal detachment and breaks without detachment. In: McPherson A (ed). New and Controversial Aspects of Retinal Detachment. Paul B Hoeber: New York, 1968, pp 164-172.

4 Morse PH, Scheie HG. Prophylactic cryoretinopexy of retinal breaks. Arch Ophthalmol 1974; 92: 204-207.

5 Zweng HC. Laser photocoagulation in rhegmatogenous retinal separation. In: Pruett RC, Regan CDJ (eds). Retina Congress. Appleton-Century-Crofts: New York, 1972; 551-554

6 Gratton I, Gazocchi M, Simonini F, Fattori CM, Citroni M. Argon laser photocoagulation in the management of retinal detachment and predisposing lesions. Lasers Surg Med 1984; 4: 337-344.

7 Hyams SW, Meir E, Ivry M, Krakowski D, Barkai S, Jedwab E, Neumann E. Chorioretinal lesions predisposing to retinal detachment. Am J Ophthalmol 1974; 78: 429-437.

8 Davi SM. Natural history of retinal breaks without detachment. Arch Ophthalmol 1974; 92: 183-195.

9 Colyear BH, Pischel DK. Preventive treatment of retinal detachment by means of light coagulation. Trans Pac Coast Ophthalmol Soc 1960; 41: 193-217.

10 Smiddy WE, Flynn HW, Nicholson DH, Clarkson JG, Gass JD, Olsen KR, Feuer W. Results and complications in treated retinal breaks. Am J Ophthalmol 1991; 112(6): 623-630.

11 Sigelman J. Vitreous base classification of retinal tears: clinical application. Surv Ophthalmol 1980; 25: 59-74.

12 Mcpherson A, O'Malley R, Beltangrady SS. Management of the fellow eyes of patients with rhegmatogenous retinal detachment. Ophthalmology 1981; 88: 922.

13 Folk JL, Arriendale L, Klugman MR. The fellow eye of patients with phakic lattice retinal detachment. Ophthalmology 1989; 96: 72.

14 Pollak A, Oliver M. Argon laser photocoagulation of symptomatic flap tears and retinal breaks of fellow eyes. Br J Ophthalmol 1981; 65: 469-472.

15 Preferred Practice Pattern. Posterior Vitreous Detachment, Retinal Breaks, and Lattice Degeneration. American Academy of Ophthalmology: San Francisco, 2003. 